\title{
Prader-Willi syndrome and autism spectrum disorders: an evolving story
}

\author{
Elisabeth M. Dykens $\cdot$ Evon Lee $\cdot$ Elizabeth Roof
}

Received: 7 March 2011 / Accepted: 26 July 2011 /Published online: 20 August 2011

(C) Springer Science+Business Media, LLC 2011

\begin{abstract}
Prader-Willi syndrome (PWS) is well-known for its genetic and phenotypic complexities. Caused by a lack of paternally derived imprinted material on chromosome 15q11-q13, individuals with PWS have mild to moderate intellectual disabilities, repetitive and compulsive behaviors, skin picking, tantrums, irritability, hyperphagia, and increased risks of obesity. Many individuals also have cooccurring autism spectrum disorders (ASDs), psychosis, and mood disorders. Although the PWS 15q11-q13 region confers risks for autism, relatively few studies have assessed autism symptoms in PWS or directly compared social, behavioral, and cognitive functioning across groups with autism or PWS. This article identifies areas of phenotypic overlap and difference between PWS and ASD in core autism symptoms and in such comorbidities as psychiatric disorders, and dysregulated sleep and eating. Though future studies are needed, PWS provides a promising alternative lens into specific symptoms and comorbidities of autism.
\end{abstract}

Keywords Prader-Willi syndrome - Chromosome 15q11q13 $\cdot$ Autism $\cdot$ Psychosis

First described over 50 years ago, Prader-Willi syndrome (PWS) is well-known for its genetic and phenotypic complexities. Seen in approximately one in 10,000 births, PWS is caused by a lack of paternally derived imprinted material on chromosome 15q11-q13, either through paternal deletion or maternal uniparental disomy (mUPD; when both copies of chromosome 15

E. M. Dykens $(\bowtie) \cdot$ E. Lee $\cdot$ E. Roof

Departments of Psychology and Human Development, Pediatrics and Psychiatry, Vanderbilt University, Vanderbilt Kennedy Center, Nashville, TN, USA

e-mail: elisabeth.dykens@vanderbilt.edu are maternally inherited). PWS is characterized by mild to moderate levels of intellectual disability, compulsive behaviors, skinpicking, tantrums, irritability, hoarding, heightened fascination and skills with jigsaw and word search puzzles, growth hormone dysregulation, hyperphagia, and increased risks of morbid obesity. Psychosis and autism spectrum disorders are also commonly seen (Dykens and Cassidy 2003).

Despite increased genetic understandings of the $15 \mathrm{q} 11-$ q13 region (Hogart et al. 2010), PWS remains a lifethreatening and clinically challenging disorder. Management challenges in PWS stem from food-seeking behaviors combined with high rates of social, emotional, and behavioral dysfunction. Many families find that even though dietary restrictions and food-seeking behaviors require their constant attention, food issues pale in comparison to their child's tantrums, skin picking, compulsivity, and needs for sameness (Dykens et al. 2007). While hyperphagia and skin picking are highly characteristic of PWS, compulsivity and tantrums are often seen in other developmental disabilities, including autism spectrum disorders.

This article reviews similarities and differences between PWS and autism spectrum disorder (ASD), and the important role that development plays in these comparisons. As PWS entails high risks of mood disorders and psychosis, links to autism may not be as straightforward as initially thought. While PWS is one of several genetic disorders associated with autism, this co-occurrence is much less well-researched than autism in the context of fragile X, 22q deletion, tuberous sclerosis, Rett, SmithLemi-Optiz, or other genetic conditions (for reviews, see Betancur 2011; Grafodatskaya et al. 2010). As a group, however, so-called "syndromic autism" accounts for up to $10 \%$ of all cases with autism (e.g., Herman et al. 2007). The molecular genetic causes and behavioral phenotypes of these syndromes offer promising alternative windows into 
the mechanisms and range of phenotypic expression in ASDs (Dykens et al. 2004). Although autism-specific data in PWS are scant relative to other syndromes, this article highlights intriguing similarities and differences between these disorders and promising areas for future research.

\section{PWS and autism: genetic links and prevalence}

PWS is caused by a lack of paternally derived imprinted material on 15q11-q13, while Angelman syndrome (AS), its oppositely imprinted genetic "sister," is caused by disruptions in the maternally expressed ubiquitin-protein ligase E3A (UBE3A) gene in this same 15q11-q13 region. PWS and AS are phenotypically distinct: those with AS have an ataxic gait, severe intellectual disabilities, seizures, frequent laughter, and limited speech. Together, PWS and AS made molecular genetic history as the first disorders to show the effects of parent-of-origin genomic imprinting.

Approximately $70 \%$ of PWS cases are due to a paternal deletion at 15q11-q13. Such deletions can be further characterized according to size, with type I deletions (BP3-BP1) being about 500 bp larger than type II deletions (BP 3-BP2). Chai et al. (2003) identified four nonimprinted genes that are deleted in type I cases, but present in those with smaller, type II deletions-cytoplasmic FMR1 interacting protein 1 (CYFIP1), NIPA1, NIPA2, and GCP5. At least $10 \%$ of deletion cases have unique breakpoints that are only now being identified through high-resolution array and other techniques (Kim et al. 2007), and these individuals may ultimately facilitate genotype-phenotype linkages. About $25 \%$ of PWS cases are due to mUPD or when both copies of chromosome 15 are maternally inherited and $5 \%$ of cases to translocations or imprinting center mutations.

The PWS critical region includes a small nucleolar RNA (snoRNA) and HBII-52 (SNORD115), which has been shown to regulate the processing of the mRNA of the serotonin $2 \mathrm{C}$ receptor, located elsewhere on the genome (Kishore and Stamm 2006). New mouse models of PWS are based on this finding, including mice deleted for HBII52 (Ding et al. 2008) or with altered $2 \mathrm{C}$ receptor mRNA functioning (Morabito et al. 2010). As with other PWS models, these mice are hyperphagic but not obese. Sahoo et al. (2008) reported a boy with features consistent with the diagnosis of PWS who had a small deletion of only the snoRNA HBII-85 and part of SNORD115 region, strongly implicating these clusters in the PWS phenotype. Lack of HBII-52 expression in PWS may lead to altered processing of serotonin $2 \mathrm{C}$ receptor mRNA, which may impact the efficacy of serotonin-altering medications in this population (Dykens and Shah 2003). Although altered serotonergic functioning has long been implicated in autism, including high levels of platelet serotonin (Veenstra-Vanderweele et al. 2009), serotonin levels or serotonin-altering genes have yet to be well-studied in PWS (although see Dykens et al. 2011).

The PWS/AS 15q11-q13 region is an epigenetic "hotspot" for ASD susceptibility genes. PWS individuals with mUPD are at higher risk for ASD due to the duplication and overexpression of maternally expressed genes in the 15q11-q13 region, including UBE3A (Schanen 2006; Wassink and Piven 2000). Persons with isodicentric 15 syndrome are also at high risk for autism and maternally inherited duplications of the $15 \mathrm{q} 11-\mathrm{q} 13$ region are relatively frequent in idiopathic autism, seen in $1-3 \%$ of these cases (e.g., Bolton et al. 2001; Vortsman et al. 2006).

In a review of published studies on autism symptoms in PWS or AS, Veltman et al. (2005) concluded that $38 \%$ of children with PWS due to mUPD had co-occurring ASD compared to $18 \%$ of PWS deletion cases and $2 \%$ of AS cases. However, the pool of studies included in this review was small (nine pertained to PWS, three to AS), and studies also differed in sample size, participant ascertainment, and the assessment tools used to make autism diagnoses. Administering the Autism Screening Questionnaire to parents of 63 offspring with PWS aged 4-48 years, Veltman et al. (2005) found that $36.5 \%$ scored above the ASQ criteria for ASD with $12.7 \%$ meeting more strict criteria for autism. Although rates of autism did not differ across genetic subtypes, those with mUPD versus deletions had more autism symptoms, primarily in the social interaction domain. Table 1 illustrates these symptoms in a brief case example of a boy with PWS and ASD who was evaluated in our research program.

Also relying on parent informants, Descheemaeker et al. (2006) found that, compared to 59 controls with intellectual disabilities (ID), 59 participants with PWS showed elevated scores on the Pervasive Developmental Disorder-Mental Retardation (PDDMR) questionnaire. Rates of pervasive developmental disorder - not otherwise specified, however, were similar across the ID and PWS groups (19\% versus $15 \%$, respectively). The PDDMR primarily assesses repetitive behavior, stereotypies, and restricted interests, with few items pertaining to social impairments. Even so, those with mUPD were significantly more likely to be rated as a "loner among peers", which hints at this group's broader social problems.

More rigorous diagnostic studies have been conducted in AS. Peters et al. (2004) administered standardized autism evaluations to 19 children with AS and found that $42 \%$ met criteria for ASD on both the Autism Diagnostic Observation Schedule (ADOS) and Autism Diagnostic Interview, Revised (ADI-R). These findings contrast with the low rate of ASD in AS noted in Veltman et al. (2005), perhaps because Peters et al. (2004) used well-accepted procedures for making reliable ASD diagnoses. Subsequent studies 
Table 1 Case example of PWS mUPD and ASD

BN, a 7-year-old boy with PWS associated with mUPD, lives at home with his parents, three siblings, and attends a regular elementary school. BN has low average cognitive abilities (FSIQ=86), good health, and has been on growth hormone treatment for several years. Relative to others with PWS, BN has a low drive for food. His body mass index is in the average range. During his research visit, BN was extremely active and asked incessant questions about staff and the assessment procedures.

Standardized questionnaires and parental interviews raised strong suspicions of autism, and BN met cut-off criteria for ASD on the Autism Diagnostic Observation Schedule (ADOS Module 3); this diagnosis was confirmed after a review of his developmental and medical history. Key features on BN's ADOS included stereotyped language and inability for reciprocal conversation, lack of friends, side object gazing, hand and finger mannerisms and unusual body posturing that increased when BN became excited. BN sniffed several test items and tended to ask the same question again and again, or to repeat odd phrases, e.g., a list of children at school, "the most beautiful thing" in response to pictures in the test materials. BN had to be redirected to complete many tasks and seemed to be easily distracted and overstimulated. He was somewhat concrete but oriented to place, person, and time.

BN's parents conveyed many concerns regarding his poor interactions with classmates and his school performance in general. BN became easily overwhelmed with classroom activities and would either "shut down" or cry daily. Based on his ASD diagnosis, BN was assigned a classroom aide and, with this support, is now doing considerably better at school.

find that approximately $48 \%$ of children with AS have ASD, but with a higher frequency $(80 \%)$ in cases with type 1 deletions (Sahoo et al. 2006).

In brief, studies to date across various $15 q 11-q 13$ syndromes indicate that autism spectrum disorders occur in: the majority of persons with isodicentric 15 syndrome, approximately $40 \%$ of those with PWS due to mUPD, $15-$ $18 \%$ of PWS cases due to deletions, and $40 \%$ of those with AS, primarily those with type 1 deletions. Building on these global diagnostic observations, research now needs to delve deeper into specific autism symptoms in PWS and address such questions as:

- Do infants with PWS show the same warning signs of autism that are reliably established in young children with idiopathic autism?

- What are the specific social, communicative, and behavioral impairments in PWS that lead to suspicions of autism in the first place?

- How do risk factors or clinical presentations of autism in the $20 \%$ of paternal deletion cases compare to $40 \%$ with mUPD?

- How do autism findings relate to the high rates of psychosis, mood disorders, and severe psychiatric illness in those with mUPD?

As discussed below, data that address these questions are limited but have the potential to shed new light on the range of phenotypic expression in both autism and PWS.

\section{Features of autism in PWS}

Studies on psychopathology in PWS primarily report psychiatric diagnoses, yet these diagnoses are often problematic. Diagnostic conventions and practices differ across countries, disciplines, and classification systems, with diagnostic criteria in PWS studies ranging from International Classification of Diseases or Diagnostic and
Statistical Manual of Mental Disorders, Fourth EditionRevised to screeners, checklists, parent reports, and symptoms in medical records. Due to their communicative or cognitive delays, reliable psychiatric diagnoses are also difficult to establish in people with intellectual disabilities, including PWS (Dykens 2000). Studies are thus needed that move beyond psychiatric diagnoses and provide detailed characterizations of symptoms in PWS and the neural or physiologic processes associated with these symptoms. This need for more nuanced data becomes strikingly clear in efforts to link PWS and ASD.

Autism spectrum disorders involve impairments in social interaction, language and communication, and restricted, repetitive, and stereotyped behaviors. Of these, impaired social interactions are often considered central to the autism diagnosis and phenotype. Ironically, social dysfunction is not at all well-studied or understood in PWS. In contrast, restricted and repetitive behaviors are seen in multiple developmental disorders (Moss et al. 2009) and have been carefully examined in PWS. As such, repetitive behaviors as opposed to social concerns have served as the launching pad for work linking autism and PWS.

Repetitive and restrictive behaviors

Types of compulsive behaviors Repetitive and restrictive behaviors include highly frequent, inappropriate, and invariant behaviors that are often seen in people with a variety of intellectual and developmental disabilities. Persons with ASD manifest both stereotypies and restrictive, repetitive behaviors (Lam and Aman 2007), while those with PWS only occasionally have stereotypies and instead show pronounced repetitive, compulsive behaviors (Dykens et al. 1996; Clarke et al. 2002).

Frequent repetitive behaviors in PWS are shown in Table 2, based on 248 individuals with PWS aged 4-52 years who have participated in our research program. These frequency data were gleaned from the child and adult versions of the 
Table 2 Frequency of repetitive, compulsive-like behaviors in 248 individuals with Prader-Will syndrome

\begin{tabular}{ll} 
Compulsive-like behaviors & $(\%)$ \\
Skin picking & 80 \\
Need to tell, ask, say & 77 \\
Hoarding & 60 \\
Ordering, arranging & 41 \\
Symmetry, exactness & 41 \\
Ritualized eating & 34 \\
Reread, rewrites & 30 \\
Fearful losing things & 29 \\
Repeated checking & 24 \\
Touch, tap, rub & 21 \\
Excessive washing & 20 \\
Rectal picking & 16 \\
Repeats routines & 15 \\
Pulls hair out & 13 \\
\hline
\end{tabular}

Yale-Brown Obsessive Compulsive Scale, with some modifications to reflect PWS-specific concerns (e.g., skin picking). The functionality of these compulsive symptoms is not well understood and may vary within and across individuals with PWS. Given their less well-developed insight, it is challenging to assess the extent to which compulsions in PWS serve a similar anxiety reducing role as seen in patients with classic obsessive-compulsive disorder (OCD). Some behaviors (e.g., sorting through things) may bring enjoyment, and stopping or preventing compulsive behaviors typically leads to varying levels of distress. These behaviors appear both internally driven and responsive to environmental triggers and have been attributed to dysfunctional dopaminergic and serotonergic functioning (Dykens and Shah 2003). As discussed below, severity of compulsions, as indexed by time spent engaged in compulsions and symptom-related distress and adaptive impairment, varies across age, intelligence quotient (IQ), and PWS genetic subtype.

Beyond these compulsions, narrow interests are also seen in people with PWS, and these are not limited to food. In light of their hyperphagia and feeling "always hungry, never full", people with PWS are often preoccupied with food seeking, eating, and their next meal. Food seeking, however, lessens considerably once food sources are secured, externally controlled, and meals are predictable and routine. Importantly, people with PWS have a range of other, nonfood interests and these are quite similar to those seen in autism (e.g., movie or cartoon characters, trains, specific animals, events). Unlike those with autism, individuals with PWS are also quite likely to hoard objects or items that reflect these narrow interests (e.g., pictures, menus, cards, movies, and magazines) and typically know right away if others have tried to clean or discard these items.
Comparative studies Using a parent informant measure, the Childhood Routines Inventory, Greaves et al. (2006) compared repetitive behavior in 80 children with PWS versus 89 with ASD. The two groups had similar scores in repetitive and "just right" domains, as well as in the intensity and frequency of these behaviors. Children with PWS were more apt to collect and store objects, while those with ASD were more likely to line up objects and be aware of such details as dust or flecks of dirt.

Moss et al. (2009) administered the Repetitive Behavior Questionnaire to parents of offspring with seven different genetic syndromes associated with IDD (Cru du Chat, Smith-Magenis, Lowe, Cornelia de Lange, fragile $\mathrm{X}$, Angelman, and Prader-Willi syndromes). Relative to other syndromic groups, those with PWS had a profile of increased compulsivity, insistence on sameness, hoarding, strong preference for routine, and low stereotypies. Previous studies have also found increased compulsivity in PWS relative to those with Smith-Magenis syndrome, Down syndrome, and nonspecific intellectual disabilities (Dykens and Kasari 1997), as well as to those with intellectual disabilities matched on degree of obesity (Clarke et al. 2002). Dykens et al. (1996) compared Yale-Brown Obsessive Compulsive Scale scores across 43 adults with PWS to 43 patients with obsessive-compulsive disorder. The number and severity of compulsive behaviors were similar across groups, although those with PWS were more likely to hoard, while those with OCD were more likely to check.

Correlates of compulsivity Correlates of repetitive, compulsive behaviors in PWS have also been identified. Gender differences, for example, are not generally found in these behaviors, although increased severity of compulsive symptoms have occasionally been reported in males (Clarke et al. 2002; Dykens et al. 1996). Level of IQ is negatively correlated with repetitive behaviors in PWS, such that those with lower IQs show more pronounced compulsivity, a finding also seen in those with intellectual disabilities in general (Dykens et al. 1996; Clarke et al. 2002; Holland et al. 2003).

Data are contradictory regarding the role that PWS genetic subtypes play in repetitive, compulsive-like behaviors. Aside from somewhat lower skin picking in mUPD cases (Symons et al. 1999; Dykens and Roof 2008), researchers report inconsistent differences in repetitive behavior across genetic subtypes. Some groups find more cleaning and redoing compulsions (and lower adaptive and academic skills) in people with type I deletions (Butler et al. 2004; Hartley et al. 2005, Zarcone et al. 2007), while other do not (Milner et al. 2005; Varela et al. 2005). Inconsistent findings may be due to small sample sizes (e.g., Butler et al. 2004; deletion, $n=24$ ) and the diverse age ranges of participants. 
In the largest cohort studied to date, with 55 deletion and 33 mUPD cases, Dykens and Roof (2008) found behavioral differences across genetic subtypes only in relation to age. Age-related increases were seen in obsessional thinking and hoarding in those with mUPD, which is consistent with their vulnerabilities to psychiatric illnesses in young adulthood. In those with type II deletions, no relations were found between age and compulsive or other behaviors. In contrast to both of these genetic subtypes, those with type I deletions showed consistent, negative associations between age and compulsivity, externalizing problems, adaptive behavior skills, and hyperphagia. In essence, although their problem behaviors appear to mellow over time, they may also show less engagement and activities in general.

It is unknown why the group with type I deletions showed age-related mellowing, but a reasonable explanation involves those genes that are deleted in type I individuals but present in others with PWS. Of these, CYFIP1 is of particular interest, as it has been linked to other $15 \mathrm{q} 11-\mathrm{q} 13$ disorders and to an unusual variant of fragile $\mathrm{X}$ syndrome. CYFIP1 is a primary target of fragile $\mathrm{X}$ mental retardation protein (FMRP), the protein involved in fragile X syndrome, and likely enables FMRP to carry out its functions in transporting and regulating mRNAs (Chai et al. 2003). Nishimura et al. (2007) reported that CYFIP1 was selectively over-expressed in cases with maternal duplications of $15 \mathrm{q} 11-\mathrm{q} 13$ and autism. As excess CYFIP1 was also found in fragile $\mathrm{X}$ cases and autism, Nishimura et al. (2007) propose that CYFIP1 is a common molecular link between co-occurring fragile $\mathrm{X}$ syndrome and ASD, and $15 \mathrm{q}$ duplications and ASD. Using a different cohort, Nowicki et al. (2007) found altered CYFIP1 in 13 individuals with fragile $\mathrm{X}$ syndrome and a Prader-Willi phenotype. These atypical cases had fragile $\mathrm{X}$ mutations, along with obesity, hyperphagia, compulsivity, symptoms of ASD, and other sporadic PWS features. Compared to a group with classic fragile $\mathrm{X}$ syndrome or typical controls, these fragile X-PWS phenotype cases had reduced levels of CYFIP1 mRNA. Additional studies are needed on the role that CYFIP1 and other non-imprinted genes play in the expression and trajectory of compulsivity and other behaviors in PWS.

Self-injurious behaviors Self-injurious behaviors (SIBs) are often studied alongside repetitive behaviors and stereotypies. Although people with PWS or autism have high rates of SIBs, research has not directly compared the types, topologies, or severity of SIBs across these two disorders. The majority of those with PWS (75-85\%) engage in skin picking (Dykens et al. 1999; Symons et al. 1999) and in some individuals picking may lead to recurring infections and serious medical complications. Rectal picking also occurs in about $20 \%$, though this may be an underestimate as parents or individuals are reluctant to spontaneously report this behavior (Dykens, unpublished data). Skin picking may lessen with age, is not related to IQ, and is slightly less common in those with mUPD. Neither behavioral nor pharmacological treatments have succeeded in consistently reducing skin picking in PWS.

In contrast, those with ASD show more diverse forms of SIBs (e.g., biting and head banging); and compared to others with intellectual or developmental disabilities, those with autism invariably show more frequent and severe SIBs (e.g., Bodfish et al. 2000; Oliver and Richards 2010). SIBs in autism are inversely related to IQ and may lessen with age, and other features of SIBs have also recently been identified. Relating repetitive behaviors and SIBs in children with autism to their levels of whole blood serotonin, Kolevzon et al. (2010) found an inverse relation between serotonin and SIBs but not to repetitive behaviors. The specificity of serotonin to SIBs is interesting, as previous studies have not generally found associations between plasma serotonin and behavioral functioning in autism (see Veenstra-VanderWeele et al. 2009). Focusing on adults, Cohen et al. (2010) found that men with autism were more likely to exhibit aggression aimed at others, while women with autism were much more likely to engage in SIBs. Given the preponderance of males with autism, females are less likely to be studied, and apparent gender differences in SIBs underscore the need for more research with females on the autism spectrum.

In brief, PWS and ASD involve both similarities and differences in their profiles of characteristic repetitive and self-injurious behaviors. Even against a shared backdrop of insistence on sameness, repetitive questioning, and narrow interests, PWS is distinguished by hoarding and skin picking, and autism by stereotypies and more diverse and severe SIBs. Further research is needed on the underlying neurobiology of these behaviors in both PWS and autism, and on behavioral or pharmacological treatments that address these salient problems.

Social and communicative impairment

Although many persons with PWS have problems getting along with others, data are scant on social impairment, which is unfortunate given the central role that social dysfunction plays in autism. Compared to age-matched persons with Williams or Down syndromes, those with PWS have similar socialization scores on the Vineland Adaptive Behavior Scales. They also have the same numbers of friends but they see these friends less often, are less competent with them, and experience more social problems in general (Dykens and Kasari 1997; Rosner et al. 2004). Further, socially competent behaviors in PWS do not 
seem to increase with age and the lack of age-related gains in these competencies contrast with age-related gains in both Williams and Down syndromes (Rosner et al. 2004). While these data depict social problems, they do not address specific impairments that constitute the hallmark of autism in face processing, social cognition, or communication.

Aberrant face processing has been identified in people with ASD using eye tracking, event-related potentials (ERPs), and functional neuroimaging. Eye tracking studies, for example, show that children with ASD often demonstrate less fixation on eyes and/or they have a relative proficiency in processing the mouth region relative to the eye region (Dalton et al. 2005; Klin et al. 2002). Studies are now underway using eye tracking in PWS. In ERP work, the N170 is often used as an electrophysiological marker for the encoding of upright faces and children with autism appear to have a dampening of the N170 in response when viewing faces (Grice et al. 2005). A preliminary study in PWS found a similar dampening effect in eight individuals with paternal deletions (Halit et al. 2011). The eight participants with mUPD showed a more typical N170 response to faces, but their eye gaze processing was similar to adults with autism. Data suggest unusual perceptual processing of faces in both genetic subtypes of PWS.

At least 200 neuroimaging studies have examined aspects of face or emotional processing in people with autism or those at risk for ASD, including siblings. Kaiser et al. (2010) recently placed this literature into a broader conceptual framework of disrupted neural states, traits, and compensatory activities in persons with autism and their unaffected siblings. In contrast, no fMRI studies have been published of face processing in PWS. The five fMRI studies published to date in PWS have all examined various aspects of food processing, reward circuits, and satiety. Findings show marked delays in satiety and aberrant post-meal activation of circuits involved in hunger (see Dimitropoulos and Schultz 2008).

Beyond face processing, data are also scant on other aspects of social cognition in PWS. Tager-Flusberg and Sullivan (2000) found that children with PWS fared as well if not better than children with Williams syndrome or other disabilities in theory of mind tasks tapping false beliefs, social actions, and emotional recognition. Difficulties in PWS may arise, however, when tasks require an appreciation for more complex or abstract mental states. Using a social attribution task involving ambiguous stimuli (moving shapes in a video), Koenig et al. (2004) report that those with PWS performed as poorly as those with ASD and below IQ-matched controls without ASD. Participants with PWS made simple cognitive attributions about the moving shapes but were less able to make inferences about affective states related to the scenario (e.g., envy, admiration, and appreciation). They thus failed to attend to pertinent details and produce a coherent social story.
Additional research is clearly needed on social functioning in PWS, especially work that uses similar neural and behavioral methodologies as the autism literature. The lack of detailed data on social or communicative functioning in PWS limits its usefulness as a genetic model for autism, and, on a practical level, it muddies the intervention waters considerably. As autism can be identified at increasingly young ages (12-18 months), with most children optimally diagnosed by age 3 , studies are especially needed on social and communicative development in infants with PWS.

\section{Infants and young children with PWS}

Infants with PWS or ASD demonstrate developmental delays, as well as gradual changes over time in temperament, emotional regulation, and difficulties in shifting attention from food or other perseverative interests. A rich literature exists on young children suspected of having autism or who are at high risk for ASD given their sibling and familial status. This body of research has identified the sensitivity and specificity of several autism screening tools for young children, standards for best practices in making autism diagnoses in young children, the nuances of social, cognitive, and motor developments and the efficacy of different models of early intervention (see Zwaigenbaum 2010 for a review). In contrast, infants with PWS have not been studied through the same careful developmental lens, and major gaps in knowledge exist on early social and communicative functioning and indices of autism in these children. Table 3 compares key features in infants with PWS versus autism.

Infants with PWS are hypotonic, lethargic, have difficulty sucking, and often need to be awakened for feedings; one feeding can literally take hours. Children and adults with PWS also have well-documented growth hormone deficiency and growth hormone treatment is now an Food and Drug Administration-approved best practice in the management of infants and children (see Miller et al. 2006 for guidelines). The benefits of growth hormone therapy (GHT) are wellestablished and include reduced body fat and increased lean muscle mass, linear growth, agility, muscle strength, coordination, and exercise tolerance; a softening of the classic PWS facial features and in toddlers, earlier ages of walking independently (Carrel et al. 1999; Myers et al. 2007).

Anecdotally, parents report that their children on GHT are more alert, energetic, attentive, and cooperative (Carrel et al. 1999), which may lead to increased learning opportunities. Indeed, compared to untreated controls, growth hormone-treated infants tested 1 year apart showed greater gains in language, cognition, and motor functioning (Festen et al. 2008; Myers et al. 2007). It is unclear if such gains in infants or toddlers are sustained over the course of development. 
Table 3 Characteristics of infants and young children with PWS or autism spectrum disorders

\begin{tabular}{lll}
\hline & PWS & Idiopathic Autism \\
\hline Failure to thrive & + & \pm \\
Poor suck, feeding & + & \pm \\
Central hypotonia & + & \pm \\
Motor delays $^{\mathrm{a}}$ & + & \pm \\
Developmental regression & - & \pm \\
Impaired social communication $^{\mathrm{b}}$ & Limited data & + \\
Repetitive interests, behaviors $^{\mathrm{c}}$ & + & + \\
Delays in play skills $^{\mathrm{d}}$ & Limited data & +
\end{tabular}

$(+)$ indicates a consistently seen feature, $(-)$ indicates that the feature is not generally present, $( \pm)$ indicates that the feature is variably present or not consistently found

${ }^{a}$ Motor delays are less pronounced in PWS infants receiving growth hormone therapy

${ }^{\mathrm{b}}$ Social communication impairment includes: atypical eye gaze, orienting to name, social smiling, and social interest and affect, with reduced expression of positive emotion

${ }^{\mathrm{c}}$ Repetitive behaviors encompass atypical exploration of toys or objects, including prolonged visual examination, unusually repetitive actions

${ }^{\mathrm{d}}$ Delays in play skills include motor imitation and functional use of toys (summarized in Zwaigenbaum, 2010 for infants aged 1218 months)

In brief, recent studies on infants with PWS have primarily evaluated the effects of GHT, and in years past, described their failure to thrive, metabolic rates, body fat, and delayed motor and language milestones. Unlike infants with ASDs, studies have not identified trajectories of social or communicative development in infants with PWS. Clinical lore suggests that infants with PWS are pleasant, cooperative, and friendly (Cassidy et al. 2000). After this period of apparent social responsiveness, they develop notable behavior problems, including temper tantrums, stubbornness, skin picking, and eventually food seeking (Dimitropoulos et al. 2001). No data have been published that specify early social and communicative deficits in children with PWS in eye gaze, shared positive affect, and social gestures such as pointing, joint attention and orientation to name. Prospective studies are needed that examine these key indices of social-communicative functioning, including factors that discriminate infants with PWS from those who are later diagnosed with ASD.

\section{Associated features of autism or PWS}

Salient associated features in autism or PWS include cooccurring psychiatric disorders, concerns with food and obesity, difficulties with sleep, and apparent strengths in visual-spatial functioning. Although not exhaustive, these features are relatively well-studied within each disorder and suggest several intriguing avenues for future research that directly compares groups with PWS versus ASD.

\section{Psychiatric disorders}

Psychiatric studies in PWS have relied solely on diagnostic labels, leaving specific psychiatric symptoms unclear or poorly described. Examining PWS cases that screened positive for psychopathology, Soni et al. (2007) report that affective illness was more prevalent in mUPD versus deletion cases (64\% as opposed $28 \%)$. Further, they note that more severe psychiatric presentations, including bipolar illness and psychosis with or without depression, were only seen in those with mUPD. In contrast, Descheemaeker et al. (2002) followed 52 adults with PWS; and of the eight with psychiatric diagnoses, "bipolar affective disorders" were only reported in those with paternal deletions.

Examining 33 cases with mUPD, aged 5-36 years $(M=$ 19 years), we found that they were more than twice as likely as their counterparts with deletions to have been psychiatrically hospitalized (55\% versus $20 \%$; Dykens and Roof 2008). Unlike those with deletions, our mUPD sample also manifested significant, age-related increases in thought problems, noncompliant behavior, obsessions, and hoarding. These findings are consistent with previous reports of age-related increases in psychopathologies in this genetic subtype. A case example of a young adult with mUPD and co-occurring psychosis is presented in Table 4.

Explanations for high rates of psychosis in mUPD generally implicate increased expression of maternally imprinted genes, especially UBE3A. Recently, however, gamma-aminobutyric acid receptor genes located in the PWS 15q11-q13 region have also been implicated; these genes are receptors for the brain's primary inhibitory neurotransmitter. Sharp et al. (2010) recently identified a novel differentially methylated regions (DMR) in the PWS critical region that was distal to gamma-aminobutyric acid A receptor- $\gamma 3$ (GABRG3). Webb et al. (2008) previously identified this same region as conferring increased risks of psychosis in adults with PWS due to mUPD, but not paternal deletion. While other DMRs in the 15q11-q13 region showed complete maternal or paternal methylation, this GABRG3 region was only partially methylated, suggesting that imprinting at this site is weakly regulated (Sharp et al. 2010). Relatively weaker parent of origin expression may help explain previous conflicting reports about methylation at the GABRG3 site. Sharp et al. (2010) hypothesize that this DMR represents a promising candidate locus, or genetic or epigenetic variation that confers risks for psychosis in PWS, and future work may suggest ties to autism as well.

If the majority of people with mUPD develop psychosis or severe affective illness by late adolescence or early adulthood 
Table 4 Case example of PWS mUPD and psychosis

JJ, a 21-year-old male with PWS due to mUPD, lives at home with his parents and attends a vocational day program. He is healthy following a weight loss of $103 \mathrm{lb}$ at age 17 that occurred during and after a stay in an in-patient hospital specializing in PWS. At the time of his follow-up research visit, JJ weighed $136 \mathrm{lb}$, was $5 \mathrm{ft}$ tall, and taking antipsychotic medications. His parents were diligent with his diet and locking food. JJ was quite proud of his weight loss and tended to open conversations with, "I lost $103 \mathrm{lb}$, are you proud of me?"

JJ's parents reported increased agitation and aggressive behavior aimed at both them and his program staff (e.g., hitting, pushing, verbal threats). He was emotionally labile, laughing, for example, over something funny "in my head" and then within seconds being verbally abusive. At age $14 \mathrm{JJ}$ experienced auditory and visual hallucinations, including an episode when his parents found him sitting naked on a couch outside their bedroom door, seeing and talking to make believe cartoon characters. He was subsequently placed on anti-psychotic medications but continued to have looseness of thoughts and magical thinking.

During his visit to the lab, JJ hit the staff and then demanded that they repeat the phrase "No, I did not hit you", and he became enraged when they did not exactly comply. JJ's speech was repetitious, nasal, pressured and perseverative, and sprinkled with demands that the staff repeat phrases verbatim that were unrelated to context, e.g., "Yes, that really looks like blue green." JJ believed that others could know what he was thinking even if he did not say it aloud and he became angry when the staff would not acknowledge his powers and believed that they were making fun of him. He was not oriented to person or place and insisted on eating lunch at his favorite restaurant some 600 miles away.

JJ's cognitive level (FSIQ =41) had declined dramatically since his first psychotic episode at age 14 when he was functioning in the borderline range (FSIQ $=77$ ). His parents were quite concerned that his escalating and bizarre behavior would not allow him to continue in the vocational day program and they feared that they would need to quit working in order to take care of him. The research team referred JJ to a PWS residential program for evaluation and possible placement as his escalating behaviors and thought disturbances required a more intensive treatment approach.

(Boer et al. 2002; Vogels et al. 2003), then PWS due to mUPD may provide an extremely useful window into psychosis in the general population. However, the specific symptoms associated with psychosis in PWS have yet to be well-described, leaving it unknown if these episodes are characterized by visual or auditory hallucinations, cognitive distortions, magical thinking, paranoia, severe or unusual obsessions, or by withdrawal, irritability, oppositionality, and changes in sleep, appetite, or activity levels.

Using a retrospective record review of adults with mUPD and psychosis, Vogels et al. (2003) noted that most had symptoms of autism or autism diagnoses as children. This finding is puzzling, as data on secondary psychiatric conditions in people with ASD consistently find increased rates of depression and anxiety disorders, but substantially less overlap with psychosis or schizophrenia (Joshi et al. 2010; Leyfer et al. 2006; Tasatsanis 2003). Based on a psychopharmacology clinic sample of children and adolescents, Joshi et al. (2010) found that $61 \%$ of 217 patients with ASDs had multiple anxiety disorders, 83\% had attention deficit hyperactivity disorder (ADHD), and 20\% had psychosis. These rates were significantly higher than nonASD clinic patients and confirm earlier reports of elevated ADHD, anxiety and affective disorders, but not psychosis, in persons with ASD (Leyfer et al. 2006). The 20\% of ASD patients with psychosis in the Joshi et al. (2010) study were not further described. If psychosis is indeed rare in autism, then comorbidities of both autism and psychosis in PWS is quite discrepant with the general ASD population.

It is not clear how to best reconcile research indicating high rates of both autism and psychosis in PWS. Some suggest an apparent "evolution" from autism in childhood to psychosis in adulthood (Descheemaeker et al. 2002). Others assert that autism diagnoses in neurodevelopmental disorders with strong associations to schizophrenia, such as $22 \mathrm{q}$ deletion syndrome or Klineflelter's syndrome, are actually false positives (e.g., Eliez 2007). In this view, autism diagnoses are an unsuccessful or superficial effort to capture premorbid personality or social dysfunction or what is essentially a prodromal state on the road to psychosis. Still others hypothesize that autism and psychosis are diametric disorders in their clinical phenotypes, and genetic and neural underpinnings (Crespi and Badcock 2008). Crespi (2008) used disruptions in maternal imprinting and proneness to autism in AS versus disrupted paternal imprinting and proneness to psychosis in PWS to support the conflict theory of imprinting in evolutionary biology.

However, none of these possible explanations can be properly evaluated without detailed data on aberrant social, cognitive, or emotional processes in PWS. Categorical diagnoses of autism or psychosis are inadequate, and more fine-tuned and nuanced phenotypic descriptions in PWS are needed in order to address these hypotheses. An unclear symptom picture in PWS also severely limits treatment. If up to $40 \%$ of those with mUPD and $20 \%$ with deletions indeed develop autism, then they may benefit from early interventions that help children with autism in general. If the majority of persons with mUPD develop psychosis, then treatment can also move to a prevention mode, perhaps minimizing disease course via pharmacotherapy, behavioral, or other interventions. Preventative measures, however, first require identification of the symptoms being targeted and when they emerge or worsen.

Food and obesity

Although hyperphagia in PWS stands out, the drive for food in affected individuals is actually quite nuanced. 
Unlike previous views of PWS as a "two-stage" disorder (failure to thrive followed by onset of hyperphagia in early childhood), PWS is comprised of up to seven distinct nutritional stages and transitional periods that reflect different phases of food intake, underlying neuroendocrine status, and degree of obesity (Miller et al. 2011). Fullblown hyperphagia varies in length and also waxes and wanes in severity (Dykens et al. 2007). People with PWS rarely vomit or report gastrointestinal (GI) distress, yet these relatively benign complaints in most people can signal a life-threatening emergency in PWS related to acute gastric dilation, perforation, necrosis, and death (Stevenson et al. 2007). Higher risks of gastric dilation and rupture are seen in individuals with rapid weight loss or who are generally slim but have a binge-eating episode.

In contrast to hyperphagia in PWS, approximately 23\% of children and adolescents with ASD have food selectivity, including food refusal, a limited food repertoire, and high frequency of eating single foods (Bandini et al. 2010). Food selectivity in ASD may relate to GI distress, including constipation, encopresis, and diarrhea, seen in approximately $25 \%$ of children with this disorder (e.g., Joshi et al. 2010; Nikolov et al. 2009). Recent data implicate associations between the MET C polymorphism and GI symptoms in children with ASD as well as associations between MET C and social and communicative symptoms but only in those with GI difficulties (Campbell et al. 2009).

Relative to the general population, children and adolescents with ASD are more likely to be obese or overweight. Compared to typical 10-17 year olds, Chen et al. (2010) found much higher rates of obesity in children with autism (12.2\% versus $23.4 \%$, respectively). Among adults, Eaves and Ho (2008) report that $42 \%$ were overweight or obese. Risk factors for obesity in ASD are similar to those seen in other disability groups, including a sedentary lifestyle, lack of regular activities or exercise, not being able to readily access gyms or recreational sports, limited knowledge about nutrition or healthy food choices, and appetitealtering side effects of psychotropic medications. Although obesity is increasingly identified in ASD, few evidencebased, weight-reduction programs exist that take into account the unique aspects of ASD.

Complications of obesity are the leading causes of premature death in adults with PWS (e.g., Einfeld et al. 2006); and obesity in this syndrome is related to mental health in complicated ways. In the general population, being overweight or obese is often associated with low selfesteem, and losing weight and being fit and trim with improved esteem (e.g., Simon et al. 2006). People with PWS, however, seem to show the opposite pattern. Specifically, adolescents and adults with lower body mass indices have increased compulsive behaviors, hoarding, and withdrawal, and are also more nervous, tense, tearful, distressed, upset, agitated, and cognitively disorganized (Dykens 2004; Hartley et al. 2005).

Although reasons for these counterintuitive finding are unknown, they may be associated with the effort, and physiological and psychological stress, of maintaining a lower weight. Due to their hypotonia and low-resting metabolic rate, persons with PWS typically require fewer calories than others to lose or maintain weight. Chronic, very low caloric restrictions $(800-1,200 \mathrm{k} / \mathrm{cal}$ daily) or sudden weight loss may contribute to increased distress, disorganization, or compulsivity, perhaps more so in those already at risk due to mUPD. Simply put, the lifelong experience of being "always hungry, never full" may lead to more distress when access to food is curbed and weight loss or maintenance is achieved. Future studies using biomarkers of stress could shed some light on these hypotheses and inform decisions about balancing dietary restrictions with quality of life in persons with PWS and their families.

Sleep

Sleep disturbances occur in the majority of children with autism, especially sleep-onset insomnia, nocturnal awakening, and shorter overall nighttime sleep duration (Goldman et al. 2009). Examining sleep in a large cohort of children with ASD, Goldman et al. (2009) found that poor sleepers, as determined by polysomnography, actigraphy, and parent report, had more daytime behavior problems, primarily hyperactivity and repetitive behaviors, than those deemed good sleepers. Further, actigraphic measures of wakefulness after sleep onset and sleep fragmentation were correlated with hyperactivity and restrictive and repetitive behaviors.

Aberrant sleep in PWS is well-described, including high rates of central and obstructive sleep apnea, abnormal arousal, abnormal circadian rhythm in rapid eye movement (REM) sleep, night awakenings, and excessive daytime sleepiness (Maas et al. 2010; Yee et al. 2007). Obesity may worsen some of these sleep parameters, yet does not completely explain abnormal REM or excessive daytime sleepiness, which are likely related to hypothalamic dysfunction (Bruni et al. 2010). Indeed, excessive daytime sleepiness can be quite impairing for individuals, regardless of their weight. Although disrupted sleep is associated with behavior problems in people with autism and intellectual disabilities in general (see Richdale and Schreck 2009 for a review), such relations are not readily apparent in PWS (e.g., Maas et al. 2010).

Beyond behavior problems, compelling evidence from both clinical and typical populations link sleep duration and quality to specific aspects of memory and cognition (see Durmer and Dinges 2005 for a review). Even so, researchers have yet to examine relations between sleep and cognition in PWS, autism, or other disability groups. 
Persons with PWS are prone to obstructive sleep apnea (e.g., Bruni et al. 2007), a known risk factor for cognitive deficits, which further highlights the need for research on cognition, sleep, behavior, and mood in this syndrome.

\section{Visual-spatial strengths}

Relative to their verbal skills, persons with autism appear to have strengths in visual-spatial processing. Earlier work found that people with ASD performed relatively well on standardized tasks assessing visual-spatial and pattern recognition, including block design, object assembly, or the imbedded figures test. More recent studies have finetuned these observations, pointing, for example, to intact or enhanced performance on static visual features or patterns, and inferior performance on more complex and dynamic spatial memory tasks (Bertone et al. 2005).

Persons with PWS have a similar profile. Early studies indicated relative strengths in visual-spatial functioning on standardized IQ tasks (e.g., block design), and individuals with PWS were also rumored to excel at jigsaw puzzles (Holm et al. 1993). Compared to others with intellectual disabilities, those with PWS spend more time engaged with puzzles (Dykens and Rosner 1999), and they also far outperform both IQ- and age-matched controls on jigsaw puzzles (Dykens 2002). Interestingly, they have only modest relative strengths in standardized visual spatial tasks. Verdine et al. (2008) administered puzzles and tasks tapping spatial perception, mental rotation, and spatial visualization to a sample with PWS- and MA-matched controls. Although the PWS group performed relatively poorly on the spatial tasks, they performed very well on interlocking jigsaw puzzles, including achromatic puzzles. The typical control group used a visual approach to puzzle solving, while those with PWS had a pronounced, shapebased approach. Persons with autism are also rumored to excel at jigsaw puzzles, and a competency with puzzles is one of several specialized interests tapped in the ADI-R. Even so, researchers have yet to formally assess puzzle skills in this population and how they relate to broader visual-spatial functioning.

\section{Next steps for research}

Although far from exhaustive, this review highlights phenotypic similarities and differences across PWS and ASD, as well as salient knowledge gaps that need to be addressed in future research. These gaps are reflected in Table 5, which summarizes areas of phenotypic overlap and difference addressed in this review. Importantly, as few studies have directly compared participants with PWS or ASD, many features in Table 5 are "best guess" hypotheses based on findings within each disorder.
Table 5 Hypothesized similarities and differences in core symptoms and associated features of ASD and PWS

\begin{tabular}{|c|c|c|}
\hline & PWS & ASD \\
\hline Repetitive behaviors & + & + \\
\hline Symmetry/exactness & + & + \\
\hline Narrow interests (not food) & \pm & \pm \\
\hline Skin picking & + & Limited data \\
\hline Hoarding & + & Limited data \\
\hline Need for sameness & + & + \\
\hline Verbal perseveration & + & + \\
\hline Stereotypies & - & \pm \\
\hline Self-injury (not skin picking) & - & \pm \\
\hline Poor peer relations & + & + \\
\hline Impaired theory of mind & Limited data & \pm \\
\hline Poor emotional recognition & Limited data & \pm \\
\hline Aberrant face processing & Limited data & + \\
\hline Problems reading social cues & Limited data & + \\
\hline Psychosis & \pm & Limited data \\
\hline Positive symptoms & \pm & Limited data \\
\hline Negative symptoms & + & Limited data \\
\hline Anxiety disorders & + & + \\
\hline Depressive disorders & \pm & \pm \\
\hline Excessive daytime sleepiness & + & - \\
\hline Sleep-onset insomnia & - & \pm \\
\hline Nocturnal awakening & \pm & \pm \\
\hline Obstructive sleep apnea & \pm & - \\
\hline Obesity & \pm & \pm \\
\hline Hyperphagia & + & - \\
\hline Food preoccupations & \pm & - \\
\hline Limited food selectivity & - & \pm \\
\hline Eating/food rituals & \pm & \pm \\
\hline Cognitive deficits & + & \pm \\
\hline Facility w/jigsaw puzzles & \pm & Limited data \\
\hline
\end{tabular}

$(+)$ indicates a consistently seen feature, $(-)$ indicates a feature that is not generally present, $( \pm)$ indicates a feature that is variably present or more inconsistently found

Future comparative studies are needed that take several methodological considerations into account. First, researchers need to use such gold standard tools for assessing autism in PWS as the ADOS-R and the ADI-R. Even so, these brief, interview-based assessments may not necessarily capture features of PWS that could discriminate between PWS and ASD. Consistent with best practices, then, researchers need to carefully review ADOS scores along with other behavioral, developmental, and medical data in making autism diagnoses in PWS and other genetic disorders.

A second issue pertains to comparison groups. Depending on the questions under study, various combinations of groups could be compared including individuals with PWS who do versus do not have autism diagnoses and who are 
appropriately matched to individuals with autism spectrum disorders or other intellectual disabilities. While these groups help distinguish features seen in PWS versus autism, a more nuanced approach uses comparison groups that selectively target the constructs under study. The type or severity of compulsive behaviors, for example, could be variably compared across people with PWS, PWS plus ASD, ASD, obsessive-compulsive disorder, and subtypes of patients with OCD (e.g., hoarders). Social-cognitive deficits could be compared across individuals with PWS, autism, schizophrenia, and atypical psychosis including the neurobiological underpinnings of these impairments (see Sasson et al. 2011). By studying "endophenotypes", these approaches emphasize neural, genetic, hormonal, psychophysiological, or developmental processes that are shared or distinctive to these disparate conditions.

Finally, gaps in knowledge need to be filled in with an eye toward clinical relevance. Careful descriptions of aberrant social, cognitive, and neural processes are needed for future research that aims to identify how the expression of susceptibility genes in the PWS/AS region (e.g., UBE3A, snoRNAs, and GABRG3) contributes to autism, psychosis, or other challenges. These behavioral and neural discoveries are needed to make more informed and immediate decisions about intervention. For example, should pharmacotherapy or other treatments begin as soon as adolescents with PWS show even a slight worsening or subtle onset of problems in thinking or mood? What other risk or protective factors derived from the autism, schizophrenia, or intellectual disabilities literature could inform treatment in PWS? Conversely, how does PWS inform genetic and other risks for autism or psychosis in the general population? Although PWS has not been as rigorously studied as other genetic conditions associated with autism or psychosis, it holds considerable promise for shedding new light on mechanisms and treatments for these otherwise debilitating disorders.

Acknowledgments The authors are grateful to the families and individuals with Prader-Willi syndrome who have participated in our work. This work was supported by NICHD Rare Disease Consortium Grant U54 HD061222, the Vanderbilt CTSA (Vanderbilt Institute for Clinical and Translational Research), and NICHD Grants R01HD035684 and P30HD015052.

\section{References}

Bandini LH, Anderson SE, Curtin C, Cermak S, Evans EW, Scampini $\mathrm{R}$, et al. Food selectivity in children with autism spectrum disorders and typically developing children. J Pediatr. 2010;157:259-64.

Bertone A, Mottron L, Jelenic P, Faubert J. Enhanced and diminished visuo-spatial information processing in autism depends on stimulus complexity. Brain. 2005;128:2430-41.
Betancur C. Etiological heterogeneity in autism spectrum disorders: more than 100 genetic and genomic disorders and still counting. Brain Research. 2011;1380:42-77.

Bodfish JW, Symons FJ, Parker DE, Lewis M. Varieties of repetitive behavior in autism: comparisons to mental retardation. J Autism Dev Disord. 2000;30:237-43.

Boer H, Holland A, Whittington J, Butler J, Webb T, Clarke D. Psychotic illness in people with PWS due to chromosome 15 maternal uniparental disomy. Lancet. 2002;359:135.

Bolton PF, Dennis NR, Browne CE, Thomas NS, Veltman MW, Thompson RJ, et al. The phenotypic manifestations of interstitial duplications of proximal $15 \mathrm{q}$ with special references to autism spectrum disorders. Am J Med Genet. 2001;105:675-85.

Bruni O, Ferri R, Vittori E, Novelli L, Vignati M, Porfirio MC, et al. Sleep architecture and NREM alterations in children and adolescents with Asperger syndrome. Sleep. 2007;30:1577.

Bruni O, Verrillo E, Novelli A, Ferric R. Prader-Willi syndrome: sorting out the relationships between obesity, hypersomnia, and sleep apnea. Curr Op Pulm Med. 2010;16:568-73.

Butler MG, Bittel DC, Kibiryeva N, Talebizadeh Z, Thompson T. Behavioral differences among subjects with Prader-Willi syndrome and type I or type II deletion and maternal disomy. Am Acad Pedia. 2004;113:565-73.

Campbell DB, Buie TM, Winter H, Bauman M, Sutcliffe JS, Perrin JM, et al. Distinct genetic risk based on association of MET in families with co-occurring autism and gastrointestinal conditions. Pediatrics. 2009;123:1018-24.

Carrel AL, Myers SB, Whitman BY, Allen DB. Growth hormone improves body composition, fat utilization, physical strength and agility, and growth in Prader-Willi syndrome: a controlled study. J Pediatr. 1999;134:215-21.

Cassidy SB, Dykens EM, Williams CA. Prader-Willi and Angelman syndromes: sister imprinted disorders. Am J Med Genet. 2000;97:136-46.

Chai JH, Locke DP, Greally JM, Knoll JHM, Ohta T, Dunai J, et al. Identification of four highly-conserved genes between breakpoint hotspots BP1 and BP2 of the Prader-Willi/Angelman syndromes deletion region that have undergone evolutionary transposition mediated by flanking duplicons. Am J Hum Genet. 2003;73:898925.

Chen AY, Kim SE, Houtrow AJ, Newacheck DW. Prevalence of obesity among children with chronic health conditions. Obesity. 2010;18:210-3.

Clarke DJ, Boer H, Whittington J, Holland A, Butler J, Webb T. Prader-Willi syndrome, compulsive and ritualistic behaviours: the first population-based survey. Brit J Psych. 2002;180:358-62.

Cohen IL, Tsiouris JA, Flory MJ, Kim S-Y, Freedland R, Heaney G, et al. A large-scale study of the psychometric characteristics of the IBR Modified Overt Aggression Scale: findings and evidence for increased self-destructive behaviors in adult females with autism spectrum disorder. J Autism Dev Dis. 2010;40:599-609.

Crespi B. Genomic imprinting in the development and evolution of psychotic spectrum conditions. Biol Rev. 2008;83:441-93.

Crespi B, Badcock C. Psychosis and autism as diametrical disorders of the social brain. Behav Brain Sci. 2008;31:241-61.

Dalton KM, Nacewicz BM, Johnstone T, Schaefer HS, Gernsbacher MA, Goldsmith HH, et al. Gaze fixation and the neural circuitry of face processing in autism. Nat Neurosci. 2005;8:519-626.

Descheemaeker MJ, Vogels A, Govers V, Borghgraef M, Willekens D, Swillen A, et al. Prader-Willi syndrome: new insights in the behavioural and psychiatric spectrum. J Intellect Disabil Res. 2002;46:41-50.

Descheemaeker MJ, Govers V, Vermeulen P, Fryns J-P. Pervasive developmental disorders in Prader-Willi syndrome: the Leuven experience in 59 subjects and controls. Am J Med Genet A. 2006;140A:1136-42. 
Dimitropoulos A, Schultz RT. Food-related neural circuitry in PraderWilli syndrome: response to high- versus low-calorie foods. J Autism Dev Dis. 2008;38:1642-53.

Dimitropoulos A, Feurer ID, Butler MG, Thompson T. Emergence of compulsive behavior and tantrums in children with Prader-Willi syndrome. Am J Ment Retard. 2001;106:39-51.

Ding F, Hua H, Zhang S, Solomon NM, Camper S, Pinchas C, et al. SnoRNA Snord116 (Pwcr1/MBII-85) deletion causes growth deficiency and hyperphagia in mice. PLoS ONE. 2008;3(3):e1709.

Durmer JS, Dinges DF. Neurocognitive consequences of sleep deprivation. Semin Neurol. 2005;25:117-29.

Dykens EM. Psychopathology in children with intellectual disabilities. J Child Psyc Psychiat. 2000;41:407-17.

Dykens EM. Are jigsaw puzzle skills "spared" in persons with PraderWilli syndrome? J Child Psych Psychiat. 2002;43:343-52.

Dykens EM. Maladaptive and compulsive behavior in Prader-Willi syndrome: new insights from older adults. Am J Ment Retard. 2004;109:142-53.

Dykens EM, Cassidy SB. Prader-Willi and Angelman syndromes: cognitive and behavioral phenotypes. In: Fisch G, editor. Genetics and neurobehavioral disorders. Totowa, NJ: Humana; 2003. p. $133-58$.

Dykens EM, Kasari C. Maladaptive behavior in children with PraderWilli syndrome, Down syndrome, and non-specific mental retardation. Am J Ment Retard. 1997;102:228-37.

Dykens EM, Roof E. Behavior in Prader-Willi syndrome: relationships to genetic subtypes and age. J Child Psych Psychiat. 2008;49:1011-8.

Dykens EM, Rosner BA. Refining behavioral phenotypes: personality-motivation in Williams and Prader-Willi syndromes. Am J Ment Retard. 1999;104:158-69.

Dykens EM, Shah B. Psychiatric disorders in Prader-Willi syndrome: epidemiology and treatment. CNS Drugs. 2003;17:167-78.

Dykens EM, Leckman JF, Cassidy SB. Obsessions and compulsions in Prader-Willi syndrome. J Child Psych Psychiat. 1996;37:995-1002.

Dykens EM, Cassidy SB, King BH. Maladaptive behavior differences in Prader-Will syndrome due to paternal deletions versus maternal uniparental disomy. Am J Ment Retard. 1999;104:67-77.

Dykens EM, Sutcliffe JS, Levitt P. Contrasting autism and 15q11-q13 disorders: behavioral, genetic, and pathophysiological issues. Ment Retard Dev Dis Res Rev. 2004;10:284-91.

Dykens EM, Maxwell MA, Pantino E, Kossler R, Roof E. Assessment of hyperphagia in Prader-Willi syndrome. Obesity. 2007;15:1816-26.

Dykens EM, Roof E, Bittell D, Butler M. TPH2 G/T polymorphism is associated with hyperphagia, IQ and internalizing problems in Prader-Willi syndrome. J Child Psych Psychiat. 2011;52:580-7.

Eaves LC, Ho HH. Young adult outcome of autism spectrum disorders. J Autism Dev Dis. 2008;38:739-47.

Einfeld SL, Kavanagh SJ, Smith A, Evans EJ, Tonge BJ, Taffe J. Mortality in Prader-Willi syndrome. Am J Ment Retard. 2006;111:193-8.

Eliez S. Autism in children with $22 \mathrm{q} 11.2$ deletion syndrome. J Am Acad Child Adolesc Psyc. 2007;46:433-34.

Festen DA, Wevers M, Lindgren AC, Bohm B, Otten B, Wit J, et al. Mental and motor development before and during growth hormone treatment in infants and toddlers with Prader-Willi syndrome. Clin Endocrinol. 2008;68:919-25.

Goldman S, Surdyka K, Cuevas R, Adkins K, Wang L, Malow BA. Defining the sleep phenotype in children with autism. Dev Neuropsychol. 2009;34:560-73.

Grafodatskaya D, Chung B, Szatmari P, Weksberg R. Autism spectrum disorders and epigenetics. J Am Acad Child Adolesc Psyc. 2010;49:794-809.

Greaves N, Prince E, Evans DW, Charman T. Repetitive and ritualistic behaviours in children with Prader-Willi syndrome and children with autism. J Intellect Dis Res. 2006;50:92-100.
Grice SJ, Halit H, Farroni T, Baron-Cohen S, Bolton P, Johnson MH. Neural correlates of eye-gaze detection in young children with autism. Cortex. 2005;41:342-53.

Halit H, Grice SJ, Bolton P, Johnson RH. Face and gaze processing in Prader-Willi syndrome. J Neuropsychol. 2011;2:65-77.

Hartley SL, MacLean WE, Butler MG, Zarcone J, Thompson T. Maladaptive behaviors and risk factors among the genetic subtypes of Prader-Willi syndrome. Am J Med Genet. 2005;136:140-5.

Herman GE, Henninger N, Ratliff-Schaub K, Pastore M, Fitzgerald S, McBride K. Genetic testing in autism: how much is enough? Genet Med. 2007;9:268-74.

Hogart A, Wu D, LaSalle JM, Schanen C. The comorbidity of autism with the genomic disorders of chromosome 15q11.2-q13. Neurobiol Dis. 2010;38:181-91.

Holland AJ, Whittington JE, Butler J, Webb T, Boer H, Clarke D. Behavioural phenotypes associated with specific genetic disorders: evidence from a population-based study of people with Prader-Willi syndrome. Psych Med. 2003;33:141-53.

Holm VA, Cassidy SB, Butler MG, Hanchett JM, Greenswag LR, hitman BY, et al. Prader-Willi syndrome: consensus diagnostic criteria. Pediatrics. 1993;91:398-402.

Joshi G, Petty C, Wozniak J, Henin A, Fried R, Galdo M, et al. The heavy burden of psychiatric comorbidity in youth with autism spectrum disorders: a large comparative study of a psychiatrically referred population. J Autism Dev Dis. 2010;40:1361-70.

Kaiser MD, Hudac CM, Shultz S, Lee SM, Cheung C, Berken AM, et al. Neural signatures of autism. PNAS. 2010;107:21223-8.

Kim S-J, Miller JL, Schwenk KA, Hollywood CL, Kuipers PJ, Driscoll DJ (2007). Methylation-specific multiplex ligationdependent probe amplification (MS-MLPA) in Prader-Willi syndrome. Paper presented to the 29th Annual PWSA (USA) Scientific Symposium, Dallas, TX

Kishore S, Stamm S. The snoRNA HBII-52 regulates alternative splicing of the serotonin receptor 2 C. Science. 2006;311:230-2.

Klin A, Jones W, Schultz R, Volkmar F, Cohen D. Visual fixation patterns during viewing of naturalistic social situations as predictors of social competence in individuals with autism. Arch Gen Psych. 2002;59:809-16.

Koenig K, Klin A, Schultz R. Deficits in social attribution ability in Prader-Willi syndrome. J Autism Dev Dis. 2004;34:573-80.

Kolevzon A, Newcorn JH, Kryzak L, Chaplin W, Watner D, Hollander E, et al. Relationship between whole blood serotonin and repetitive behaviors in autism. Psych Res. 2010;175:274-6.

Lam KSL, Aman MG. The Repetitive Behavior Scale-Revised: independent validation in individuals with autism spectrum disorders. J Autism Dev Dis. 2007;37:855-66.

Leyfer OT, Folstein SE, Bacalman S, Davis NO, Dinh E, Morgan J, et al. Comorbid psychiatric disorders in children with autism: interview development and rates of disorders. J Autism Dev Dis. 2006;36:849-61.

Maas APM, Sinnema M, Didden R, Maaskant MA, Smits MG, Schrander-Stumpel CT, et al. Sleep disturbances and behavioural problems in adults with Prader-Willi syndrome. J Intellect Disabil Res. 2010;54:906-17.

Miller J, Silverstein J, Shuster J, Driscoll DJ, Wagner M. Short-term effects of growth hormone on sleep abnormalities in Prader-Willi syndrome. J Clin Endocrinol Metab. 2006;91:2413-7.

Miller JL, Lynn CH, Driscoll DC, Goldstone AP, Kimonis V, Dykens E, et al. Nutritional phases in Prader-Willi syndrome. Am J Med Genet. 2011;155A:1040-9.

Milner KM, Craig EE, Thompson RJ, Veltman MWM, Thomas NS, Roberts S, et al. Prader-Willi syndrome: intellectual disabilities and behavioural features by genetic subtype. J Child Psyc Psych. 2005;46:1089-96. 
Morabito M, Abbas A, Hood J, Kesterson R, Jacobs M, Kump D, et al. Mice with altered serotonin $2 \mathrm{C}$ receptor RNA editing display characteristics of Prader-Willi syndrome. Neurobiol Dis. 2010;39:169-80.

Moss J, Oliver C, Arron K, Burbidge C, Berg K. The prevalence and phenomenology of repetitive behavior in genetic syndromes. J Autism Dev Dis. 2009;39(4):572-88.

Myers SE, Whitman BY, Carrel ALL, Moerchen V, Bekx MT, Allen DB. Two years of growth hormone therapy in young children with Prader-Willi syndrome: physical and neurodevelopmental benefits. Am J Med Genet. 2007;143A:443-8.

Nikolov RN, Bearss KE, Letting J, Erickson C, Rodowski M, Aman $\mathrm{MJ}$, et al. Gastrointestinal symptoms in a sample of children with pervasive developmental disorders. J Autism Dev Dis. 2009;39:405-13.

Nishimura Y, Martin CL, Lopez AV, Spence SJ, Alveraz-Retuerto AI, Sigmans M, et al. Genome-wide expression profiling of lymphoblastoid cell lines distinguishes different forms of autism and reveals shared pathways. Hum Mol Genet. 2007;16: 1682-98.

Nowicki S, Tassone F, Ono MY, Ferranti J, Croquette MF, GoodlinJones $\mathrm{B}$, et al. The Prader-Willi phenotype of fragile $\mathrm{X}$ syndrome. J Dev Behav Pediatr. 2007;28:133-8.

Oliver C, Richards C. Self-injurious behaviour in people with intellectual disability. Curr Op Psych. 2010;23:412-6.

Peters SU, Beaudet AL, Madduri N, Bacino CA. Autism in Angelman syndrome: implications for autism research. Clin Genet. 2004;66:530-6.

Richdale AL, Schreck KA. Sleep problems in autism spectrum disorders: prevalence, nature, and possible biopsychosocial aetiologies. Sleep Med Rev. 2009;13:403-11.

Rosner BA, Hodapp R, Fidler D, Sagun J, Dykens EM. Social competence in persons with Prader-Willi, Williams and Down syndromes. J Appl Res Intellect Disabil. 2004;17:209-17.

Sahoo T, Peters SU, Madduri N, Glaze DG, German JR, Bird LM, et al. Microarray based comparative genomic hybridization testing in deletion bearing patients with Angelman syndrome: genotypephenotype correlations. J Med Genet. 2006;43:512-6.

Sahoo T, Gaudio D, German JM, Shinawi S, Peters SU, Person RE, et al. Prader-Willi phenotype caused by paternal deficiency for the HBII-85 C/D box small nucleolar RNA cluster. Nat Genet. 2008;40:719-21.

Sasson NJ, Pinkham AE, Carpenter KLH, Beliger A. The benefit of directly comparing autism and schizophrenia for revealing mechanisms of social cognitive impairment. J Neurodev Disord. 2011;3:87-100.

Schanen NC. Epigenetics of autism spectrum disorder. Hum Mol Genet. 2006;15:R138-50.

Sharp AJ, Migliavacca E, Dupre Y, Stathaki E, Sailani MR, Baumer A, et al. Methylation profiling in individuals with uniparental disomy identifies novel differentially methylated regions on chromosome 15. Genome Res. 2010;20:1271-8.

Simon GE, Von Korf M, Saunders K, Miglioretti DL, Crane PK, van Belle $\mathrm{G}$, et al. Association between obesity and psychiatric disorders in the US adult population. Arch Gen Psych. 2006;63:824-30.

Soni S, Whittington J, Holland AJ, Webb T, Maina E, Boer H, et al. The course and outcome of psychiatric illness in people with Prader-Willi syndrome: Implications for management and treatment. J Intellect Disabil Res. 2007;51:32-42.

Stevenson DA, Heinemann J, Angulo M, Butler MG, Loker J, Rupe $\mathrm{N}$, et al. Gastric rupture and necrosis in Prader-Willi syndrome. J Pediatr Gastroenterol Nutr. 2007;45:272-4.

Symons FJ, Butler MG, Sanders MD, Feurer I, Thompson T. Selfinjurious behavior and Prader-Willi syndrome: behavioral forms and bodily locations. Am J Ment Retard. 1999;104:260-9.

Tager-Flusberg H, Sullivan K. A componential view of theory of mind: evidence from Williams syndrome. Cognition. 2000;76:59-89.

Tasatsanis K. Outcome research in Asperger syndrome. Philadelphia: Saunders; 2003

Varela, M., Kok, F., Setian, N., Kim, C. and Koiffmann, C. Impact of molecular mechanisms, including deletion size, on Prader-Willi syndrome phenotype: study of 75 patients. Clin Genet. 2005;67: $47-52$.

Veenstra-VanderWeele J, Jessen TN, Thompson BJ, Carter M, Prasad $\mathrm{HC}$, Steiner JA, et al. Modeling rare gene variation to gain insight into the oldest biomarker in autism: construction of the serotonin transporterGly56Ala knock-in mouse. J Neurodev Disord. 2009;1:158-71.

Veltman MW, Craig EE, Bolton PF. Autism spectrum disorders in Prader-Willi and Angelman syndromes: a systematic review. Psychiatr Genet. 2005; 15:243-54.

Verdine B, Troseth GL, Hodapp RM, Dykens EM. Strategies and correlates of jigsaw puzzle and visual-spatial performance in persons with Prader-Willi syndrome and matched controls. Am J Ment Retard. 2008;113:343-55.

Vogels A, Matthijs G, Legius E, Devriendt K, Fryns J. Chromosome 15 maternal unipaternal disomy and psychosis in Prader-Willi syndrome. J Med Genet. 2003;40:72-3.

Vortsman JA, Staal WG, van Daalen E, van Engeland H, Hochstenbach P, Franke L. Identification of novel autism candidate regions through analysis of reported cytogenetic abnormalities associated with autism. Mol Psych. 2006;11:18-28.

Wassink TH, Piven J. The molecular genetics of autism. Curr Psych Rep. 2000;2:170-5.

Webb T, Maina E, Soni S, Whittington J, Boer H, Clarke D, et al. In search of the psychosis gene in people with Prader-Willi syndrome. Am J Med Genet. 2008;146:843-53.

Yee BJ, Buchanan PR, Mahadev S, Banerjee D, Liu PY, Phillips C, et al. Assessment of sleep and breathing in adults with Prader-Willi syndrome: a case control series. J Clin Sleep Med. 2007;3: 713-18.

Zarcone J, Napolitano D, Peterson C, Ferraioli S, Caruso-Anderson $\mathrm{M}$, Holsen $\mathrm{L}$, et al. The relationship between compulsive behavior and academic achievement across three genetic subtypes of Prader-Willi syndrome. J Int Dis Res. 2007;51:478-87.

Zwaigenbaum L. Advances in the early detection of autism. Curr Op Neuro. 2010;23:97-102. 